\title{
A Fuzzy Adaptive Control of Doubly Fed Induction Machine (DFIM)
}

\author{
A.Saidi, Lamia Youb, Farid Naceri, Sebti Belkacem \\ Uinversite Batna-2, Departement of Electrical Engineering, Batna, Algeria
}

\begin{abstract}
In this paper, we are interested in the adaptive fuzzy control a technique has been studied and applied, namely adaptive fuzzy control based on theory of Lyapunov. The system based on the stability theory is used to approximate the gains Ke and kdce to ensure the stability of the control in real time .the simulations results obtained by using Matlab environment gives that the fuzzy adaptive control more robust, also it has superior dynamics performances. The results and test of robustness will be presented.
\end{abstract}

\section{Introduction}

The asynchronous machine with double feed (DFIM) is very popular since it profit from certain advantages compared to all the other types at variable speed, sound use in the chains of electromechanical conversion as an aero generator or engine knew a spectacular growth during last years. Indeed, it converter of energy used in order to rectify-undulate the alternating currents of the rotor has a fractional nominal output nominal of that of the generator, which reduces its cost by report/ratio with concurrent topologies(Saidi and Naceri, 2016).

The DFIM is essentially non linear, due to the coupling between the flux and the electromagnetic torque. The vector control or field orientation control allows a decoupling between the torque and the flux (Payam, et al., 2010; Chafaa, et al., 2014).

The system of fuzzy logic aims to model human reasoning and thinking, the process with linguistic variables. They are very useful when the process to control with some uncertainties or unknown variables(Sundarapandian, et al., 2016; Souli, et al., 2015; Rabie, et al., 2015;Calcev, et al., 2014).

However, in order to maintain consistent execution in the presence of true uncertainties, the use of adaptive control is,in most cases, inevitable.Fuzzy adaptive control has been the subject of intensive research during the last decade (Minth, et al., 2009; Annis, et al., 2004; Chen, et al., 1999; Chih, et al., 2004).

Therefore, the combination of the study of adaptability and uncertainty has allowed researchers to derive adaptive fuzzy controllers (Chekkouri, et al., 2003; Rabie, et al., 2015).

Several adaptive fuzzy control schemes have been proposed for complex systems In these adaptive schemes, the study of stability is based on the Lyapunov approach(Fischle, et al., 1999; Yusupbekov, et al., 2016). An adaptive fuzzy control approach can be used: the direct approach is based on the adaptation mechanism.In the direct approach,the adaptation system is used to estimate the gains of the PI-fuzzy and the control law is obtained from this PI-fuzzy controller.

Despite their dynamic capabilities and their practical implementation, the first PI-fuzzy suffered from lack of stability analysis, That is to say the stability of the closed loop is not guaranteed (Sarala, et al., 2016; Furuhashi, et al., 1998; Faquir, et al., 2016). Recently, a large class of adaptive fuzzy systems, which use the fuzzy systems of Mamdani or TS was developed to control non-linear processes, and stability has been guaranteed in the sense of the Lyapunov theory.

Treatment in this paper adaptive fuzzy control based on stability theory to recalculate real-time PI-fuzzy gains.we will develop a new order strategy which is: adaptive fuzzy control based on the theory of Lyapunov.

\section{Adaptive fuzzy control based on Lyapunov theory}

Today, there are a number of studies on the stability of fuzzy systems. However,restrictive because of nonexistence tools appropriate. To do this, the fuzzy controller being non-linear,We have to do use of nonlinear methods. such as the Lyapunov method, the theory of hyper stability or the criterion of Popov.Most of these methods are quite limiting,providing only sufficient conditions for stability in a domain restricted.

For adaptive fuzzy control based on the Lyapunov theory,we follow two steps. Lapremière is devoted to the design of a PI-fuzzy controller. The second is to define the methodology for determining the gains of a fuzzy regulator based on the Lyapunov theory. The 
control applied to the Dual Power Asynchronous Machine is provided by a PI-fuzzy controller.

We proposed a new adaptive fuzzy control strategy based on the theory of Lyapunov to determine the gains of $\mathrm{K}_{\mathrm{e}}$ and $\mathrm{K}_{\mathrm{dce}}$ (normalization), which can be applied for a large class of non-linear systems. It combines the advantages of two robust techniques and which are the control by the fuzzy logic and the control adaptive(Laamayad, et al., 2012; Chen, et al., 1999).

\subsection{Control law of a PI-fuzzy controller}

We consider a nonlinear system whose dynamic equation of the command is described in the following form:

$$
T_{e m}^{*}(t)=T_{e m}^{*}(t-1)+K_{d c e} d T_{n e m}
$$

\subsection{Fuzzification}

The error between the reference signal and the process serves to act directly (the gains of the mad regulator are recalculated in real time as a function of this error and the response of the regulator itself and gains and coefficients of adaptation).The speed error denoted e (t) is defined by:

$$
e(t)=\omega_{r e f}-\omega_{r}(t)
$$

The derivative of the speed error noted $\frac{d e(t)}{d t}$ is approximated by:

$$
\frac{d e(t)}{d t}=\dot{e}(t) \approx \frac{d e(t+1)-e(t)}{T_{e}}
$$

\section{$T_{\mathrm{e}}$ : Being the sampling period.}

The error and the derivative of the error are adapted as follows:

$$
\begin{gathered}
e(t)=K_{e} e_{n}(t) \\
\frac{d e(t)}{d t}=K_{d e} \frac{d e_{n}(t)}{d t}
\end{gathered}
$$

\subsection{Inference table}

Once the fuzzy presentation stage is complete, the gains estimated by the adjustment mechanism which uses the

\begin{tabular}{|c|c|c|c|c|c|c|}
\hline \multirow{2}{*}{\multicolumn{2}{|c|}{$d \mathrm{~T}_{\text {nem }}$}} & \multicolumn{5}{|c|}{$e_{n}$} \\
\hline & & \multirow{2}{*}{\begin{tabular}{|l|} 
NB \\
NB
\end{tabular}} & \multirow{2}{*}{$\begin{array}{l}\text { NM } \\
\text { NB }\end{array}$} & \multirow{2}{*}{$\begin{array}{c}\mathbf{Z} \\
\mathrm{NM}\end{array}$} & \multirow{2}{*}{$\begin{array}{l}\text { PM } \\
\text { NVS }\end{array}$} & \multirow{2}{*}{$\begin{array}{c}\text { PB } \\
Z\end{array}$} \\
\hline \multirow{5}{*}{$\frac{d e_{n}}{d t}$} & NB & & & & & \\
\hline & $\mathbf{N M}$ & NB & NM & NVS & $\bar{Z}$ & P S \\
\hline & $\bar{Z}$ & NM & NVS & Z & PS & PM \\
\hline & PM & NVS & $\bar{Z}$ & PS & $\overline{\mathrm{PM}}$ & PB \\
\hline & PB & $\mathrm{Z}$ & PS & PM & PB & PB \\
\hline
\end{tabular}
Lyapunov theory are sent to the fuzzy regulator to construct the output $\mathrm{dT}_{\text {nem }}(\mathrm{t})$ Therefore, the fuzzy regulator rule takes the form next :

Table 1. Inference matrix of fuzzy rules.

\subsection{Deffuzification}

For defuzzification, the method of the center of gravity presented above, we obtain:

$d T_{n e m}(t)=\frac{\sum_{j=1}^{25}=1 \mu_{A 1 J}\left(e_{n}(t)\right) \mu_{A 2 J}\left(\frac{d e_{n}(t)}{d t}\right) C_{J} S_{J}}{\sum_{j=1}^{25} \mu_{A 1 J}\left(e_{n}(t)\right) \mu_{A 2 J}\left(\frac{d e_{n}(t)}{d t}\right) C_{J} S_{J}}$

\section{Schematic diagram}

To minimize the instantaneous error between the actual speed of the machine DFIM and that of the reference speed we recalculate in real time the gains $\mathrm{k}_{\mathrm{e}}$ and $\mathrm{k}_{\mathrm{dce}}$ of the premises and the consequences by the application of an adaptation algorithm of study type stability of control in the sense of Lyapunov (Laamayad, et al., 2012; Chen, et al., 1999).

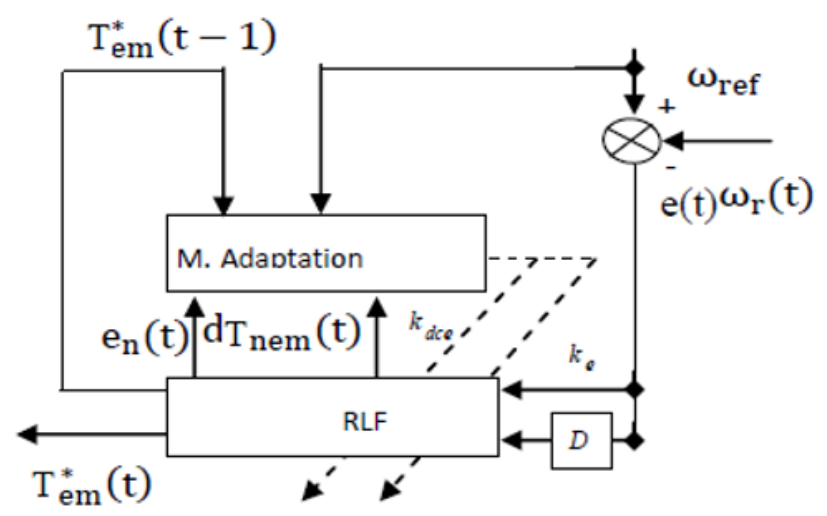

Fig 1. Structure of adaptive fuzzy control based on the Lyapunov theory.

\section{Analysis of the stability of the proposed control}

\subsection{Function of Lyapunov candidate}

Function of Lyapunov can be considered as a function of error and the variation of the gains. This is a decreasing function along the trajectories of the system. We consider a Lyapunov candidate function:

$V=\frac{1}{2}\left(e^{2}+\frac{1}{\gamma_{1}} k_{e}^{2}+\frac{1}{\gamma_{2}} k_{d c e}^{2}\right)$ 


$$
\gamma_{1}>0 \text { and } \gamma_{2}>0
$$

The derivative of the Lyapunov function with respect to real time:

$$
\frac{d V}{d t}=e \frac{d e}{d t}+\frac{k_{e}}{\gamma_{1}} \frac{d k_{e}}{d t}+\frac{k_{d c e}}{\gamma_{2}} \frac{d k_{d c e}}{d t}
$$

The following expression can be obtained:

$$
\begin{aligned}
& \frac{d V}{d t}=e\left(a_{p} \omega_{r}(t)-b_{p} T_{e m}^{*}(t-1)-b_{p} k_{d c e} d T_{n e m}(t)+d_{p} T_{r}\right)+\frac{k_{e}}{\gamma_{1}} \frac{d k_{e}}{d t} \\
& +\frac{k_{d c e}}{\gamma_{2}} \frac{d k_{d c e}}{d t}
\end{aligned}
$$

We note that we can replace the necessary expression of error e (t) by the form

next error $e(t)=k_{e} e_{n}(t)$ in the derivative of the Lyapunov function.

$$
A=\mid\left(a_{p} \omega_{r e f}-b_{p} T_{e m}^{*}(t-1) \mid\right.
$$

The gains of a fuzzy regulator are to be defined from a single necessary and sufficient condition for existence of the stability regime in the sense from Lyapunov.

$$
\frac{d V}{d t}\langle 0
$$

The inequality is verified by imposing the following adaptation law:

$$
\begin{gathered}
\frac{d k_{e}}{d t}=-\gamma_{1} e_{n} A \\
\frac{d k_{d c e}}{d t}=\gamma_{2} b_{p} k_{e} e_{n} d T_{n e m}
\end{gathered}
$$

The form of the Lyapunov function is proposed to determine the PI-fuzzy gains which ensure a convergence of the actual speed of the DFIM towards its reference value. Adaptation gains are recalculated in real time in the purpose of imposing desired stability on the controlled system that is to say the speed of adjustment at the level of the assembly

PI-fuzzy controller and the DFIM machine in the transitional regime. So, for the speed loop, the parameters of the Lyapunov function are chosen to ensure: the derivative of the

Lyapunov candidate always negative ( eq14), as well as an accuracy of speed control.

\section{Application for doubly fed induction machine}

For the adjustment of the speed of a DFIM by the adaptive fuzzy control based on the theory of lyapunov, the following method is followed:

From the reference speed and the actual speed, the adaptive fuzzy controller provides

the torque $T_{e m}^{*}(t)$. A transformation of park (dq-abc) makes it possible to calculate the currents stator of references. These currents are compared with real currents to order of each inverter.

To adjust the speed, use the output of the fuzzy controller $T_{e m}^{*}$, as well as the adjustment of gains to ensure stability of control. In our application, we used a PI-Fuzzy. The first concerns speed, where we assigned five membership functions to each entry of the model. Figure (2) illustrates the speed control structure of a doubly fed induction machine by applying the method of fuzzy adaptive control based on theory from Lyapunov.

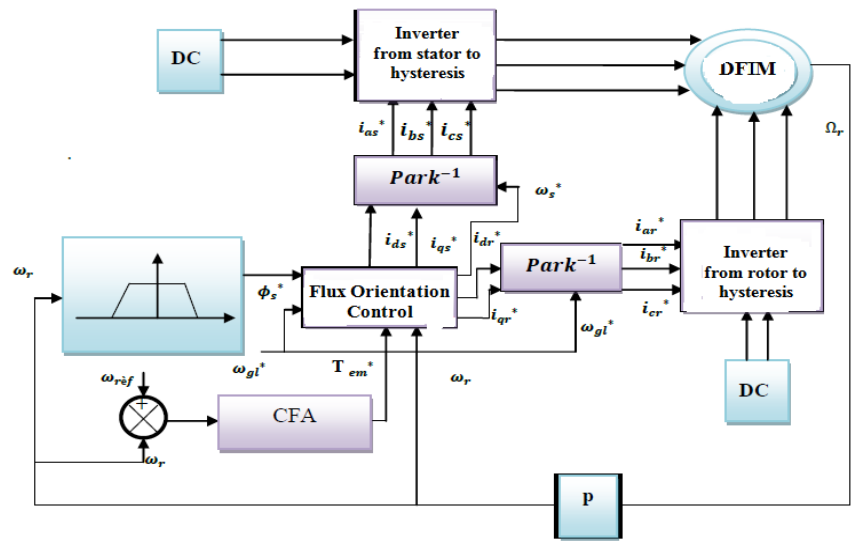

Fig 2. Structure of adaptive fuzzy control based on the Lyapunov theory

\section{Simulation results}

It appears, following the various results obtained, that the performance of speed control by the application of adaptive fuzzy control with the stability of control are very satisfactory. The coefficients $\gamma_{1}, \gamma_{2}$ have respectively 0.005 and 70 respectively. So the initial values gains $\mathrm{k}_{\mathrm{e}}, \mathrm{k}_{\mathrm{dce}}$ are respectively 7 and 5.8 , To demonstrate the adaptability of the proposed control scheme to parametric variations, we introduce parametric variations on the resistance rotor, as well as the moment of inertia at the instant $t=1 \mathrm{~s}$. Resistance and moment of inertia are increased by $150 \%$, and then the resistance and moment of inertia are decreased of $50 \%$. The responses obtained are shown in Figure (4), (5), (6) . It is clear that this parametric variation did not affect the speed control performance, which proves the 

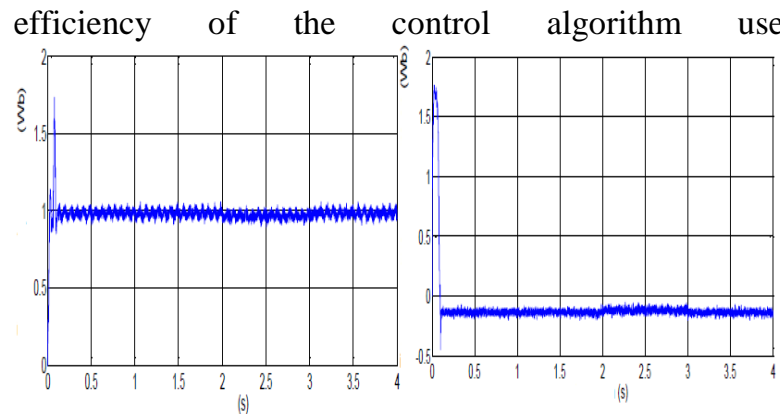

Fig (3.a). Components of direct stator flux $\varphi_{d s}$ and in quadrature
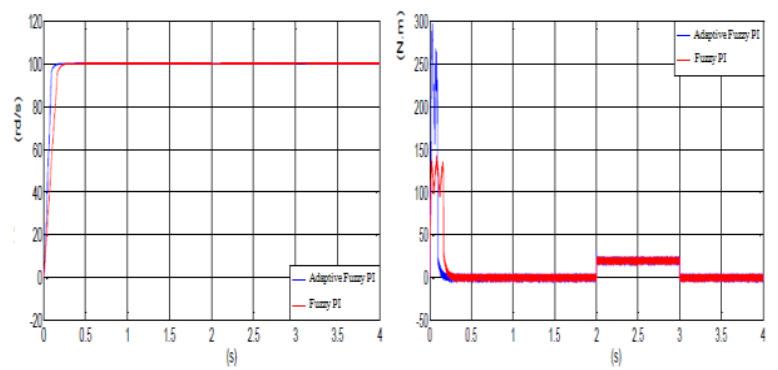

Fig (3.b). Dynamic response of speed and electromagnetic torque.

Figures (3.a) and (3.b), (3.c), show the performance of the speed control of the double-feed asynchronous machine for a set point of $100 \mathrm{rad} / \mathrm{s}$ with a variation of the load. The speed follows the reference speed despite the presence of charge. The response time is in the order of $0.1 \mathrm{~s}$. The overrun is zero
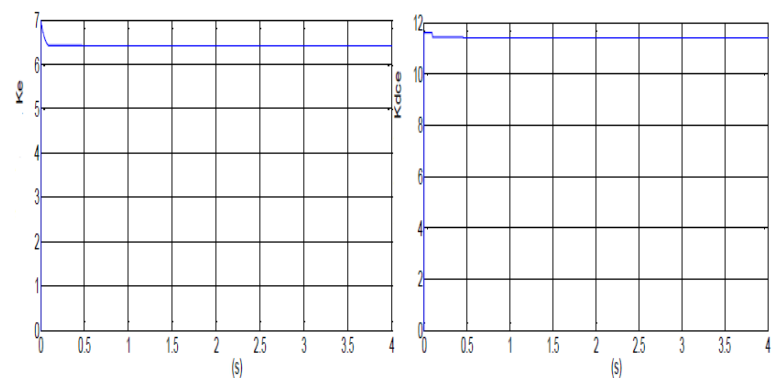

Fig (3.c). Dynamic machine response with PI-fuzzy control during the introduction of a load torque of $\left(\mathrm{T}_{1}=20\right.$ N.m) at time $\mathrm{t}=2(\mathrm{~s})$.

\subsection{Tests of Robustness}

The test consists of a variation of the rotor resistance in a range of $50 \%$ and $150 \%$. The speed and the electromagnetic torque, the stator fluxes direct and quadrature as well as the evolution of the fuzzy gains
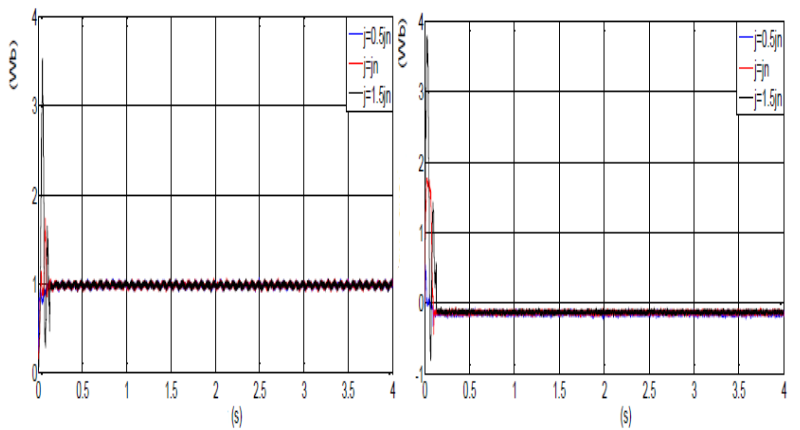

Fig (4). Components of direct stator flux $\varphi_{d s}$ and in quadrature $\varphi_{q s}$
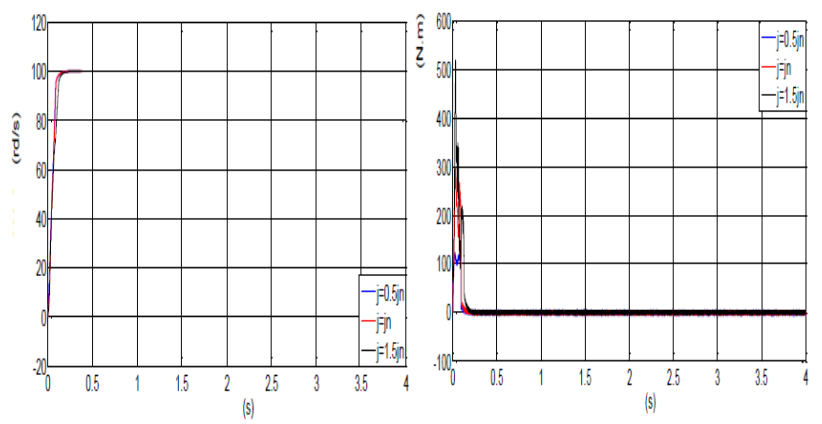

Fig (5). Dynamic response of speed and electromagnetic torque
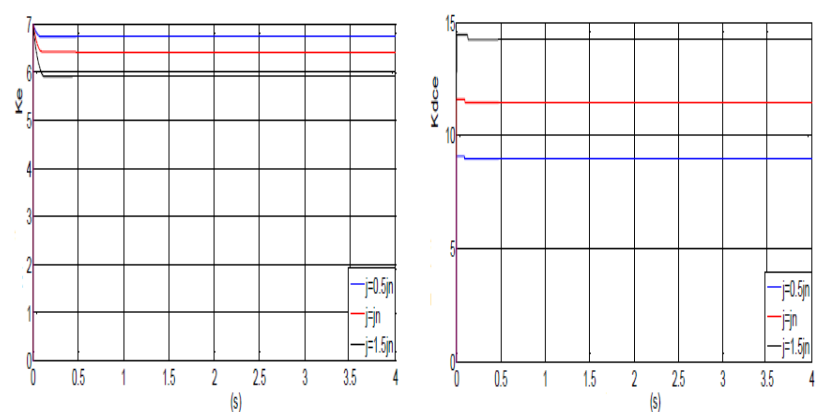

Figure (6). Evolution of the fuzzy gains $\left(\mathrm{k}_{\mathrm{e}}, \mathrm{k}_{\mathrm{dce}}\right)$

\section{Conclusion}

The obtained results as well as the various tests have shown that this technique good performance in the presence of load disturbances and reversing the direction of travel, the speed follows its reference, the stator flux follows its reference value along the axis $d$ with a zero component along the axis q. An increase in the electromagnetic torque in the presence of the resistive torque which leads to a good acceleration of the motor.

PI-fuzzy control with gain adaptation ke and kdce by the theory of Lyapunov is also tested for parametric variations of the system. The results have enabled us to judge that this new ordering strategy strong robustness in the presence of variations.

\section{References}

1. Saidi A, Naceri F. (2016), ' Comparative study between Sliding mode controller and Fuzzy Sliding 
mode controller in a speed control for doubly fed induction motor', Proceedings of IEEE International Conference on Control Engineering \& Information Technology (CEIT) Tunisia , pp. 1-6.

2. Payam F, Kashiha H. (2010), 'Robust Speed Sensorless Control of Doubly-fed Induction Machine Based on Input-output Feedback Linearization Control Using a Sliding-mode Observer ', World Appl. Sci. J, 10(11): pp13921400

3. Chafaa $\mathrm{Kh}$, Salah Khireddine $\mathrm{M}$, Slimane N , Ghanai M (2014), 'Fuzzy estimation based on type-2 fuzzy logic for adaptive Control', Proceedings of IEEE International Conference on Computer Applications \& Research (WSCAR), Sousse, Tunisia.

4. Sundarapandian V, Taher Azar A. (2016), ' TakagiSugeno fuzzy logic controller for Liu-Chen fourscroll chaotic system', Int. J. of Intelligent Engineering Informatics, Vol.4, No.2, pp.135 - 150 .

5. H.F. Ho, K.W.E. Cheng. (2009) , 'Position Control of Induction Motor using Indirect Adaptive Fuzzy Sliding Mode Control', IEEE 3rd International Conference on Power Electronics Systems and Applications, pp. 1 - 5 .

6. Souli R, bali J,Minyar Sassi Hidri. (2015) , ' A cloud-based optimal fuzzy clustering of distributed data', Int. J. of Intelligent Engineering Informatics, Vol.3, No.1, pp.42 - 56.

7. Rabie A. Ramadan. (2017) , ' Fuzzy brain storming optimisation algorithm', Int. J. of Intelligent Engineering Informatics, Vol.5, No.1, pp.67 - 79

8. Calcev G ,Greco C , Bona B (1994), ‘ Fuzzy Controller Synthesis Using Sliding Mode Approach, 'NAFIPS/IFIS/NASA'94 Proceeding of the First International Joint Conference of the North American Fuzzy Information Processing Society Biannual Conference. The Industrial Fuzzy Control and Intelligent Systems Conference, and the NASA Joint Technology , IEEE,pp.255-258 ,18-21 Dec .

9. Minth. TA CAO. (1997), 'Commande numérique de machines asynchrones par logique floue', thèse de Philosophie doctorat faculté des études supérieure de l'université Laval .

10. Annis Y, Karnavas L, Demetrios P. Papadopoulos .(2004), ' Excitation Control of a Synchronous Machine Using Polynomial Neural Networks ', Journal of Electrical Engineering, Vol. 55, No. 7-8, 169-179.

11. Chen B, Yingbi Zhang, J.Yen, Wei Zhao . (1999), 'Fuzzy Adaptive Connection Admission Control for Real-time Applications In ATM-Based Heterogeneous Networks', Journal of Intelligent and Fuzzy Systems $7,125-135$.

12. Chih L -Min and Chun-Fei H. (2004), 'Adaptive Fuzzy Sliding-Mode Control for Induction Servomotor Systems', IEEE Transactions On Energy Conversion, Vol. 19, No. 2, pp.362-368.

13. Chekkouri M, Catalài J Lopez, Rubira E, Martinez L (2003), ' Fuzzy Adaptive Control of an Induction Motor Drive' , Automatika 44, 113-122.

14. Rabie A. Ramadan, Basant R. El Samadony; Nevin M. Darwish . (2015), ' Fuzzy-based centroid localisation in WSNs', Int. J. of Intelligent Engineering Informatics, 2015 Vol.3, No.4, pp.293 -312 .

15. Fischle K and Schroder D 'An Improved Stable Adaptive Fuzzy Control Method'(1999), IEEE Trans on Fuzzy Systems, Vol.7,No.1,27

16. Yusupbekov NR, Marakhimov AR, Igamberdiev HZ , Umarov ShX . (2016), ' An Adaptive FuzzyLogic Traffic Control System in Conditions of Saturated Transport Stream', The Scientific World Journal. 